Tu SP4 15

\title{
Analysis of Channels Using Acoustic Impedance and Spectral Decomposition within Cenomanian- Turonian Reservoir in Iran
}

\author{
M.S. Mirkamali (Sahand University of Technology), N. Shad Manaman \\ (Sahand University of Technology), I. Abdollahie Fard (National Iranian Oil \\ Company), N. Keshavarz Farajkhah (Research Institute of petroleum \\ Industry), H. Khoshdel (National Iranian Oil Company) \& A.H. \\ Najafabadipour* (University of Tehran)
}

\section{SUMMARY}

Channel patterns are studied to detect subtle changes and to determine reservoir characteristics within Cenomanian-Turonian carbonate reservoir in an oil field located in the SW of Iran. The CenomanianTuronian carbonate reservoir consists of several buried channels which would certainly be interesting candidate to verify probable places of hydrocarbon accumulation in the SW of Iran. In this study, firstly iso-frequency maps are estimated using FFT method, and then acoustic impedance is obtained based on seismic inversion. The iso-frequency maps can help to clarify the channel patterns through observed variations of the channel frequency content. The opposite behaviour of the acoustic impedance is confirmed to determine reservoir characteristics using the seismic inversion method. Based on the obtained results, the channel patterns can be classified into two types in several stratigraphic levels of the Cenomanian-Turonian carbonate reservoir based on the different frequency content and the acoustic impedance from their surroundings. Finally, integrated analysis of the iso-frequency maps with the acoustic impedance maps reveals porous parts of the channel patterns with more detail than single analysis of the acoustic impedance maps. 


\section{Introduction}

Channels are critical interest of stratigraphic sequence studies in development and production for successful reservoir management of the petroleum system. The Cenomanian-Turonian carbonate setting is accompanied with presence of a complex channel deposition system in the SW of Iran (Hasanzadeh et al., 2009). Studying channels provides a better view of the region's petroleum system, due to economical importance of oil fields with the carbonate reservoir in the SW of Iran. Since channel thickness is inconsiderable compared to other geological features on the original seismic data, utilizing time-frequency methods as well as displays derived from these methods, can result in better detection of channels. Spectral decomposition of a seismic signal implies to characterize various timefrequency responses of geologic features. Among methods of the spectral decomposition, in fast Fourier transform (FFT), application of a fixed length analysis window can be provided to compare which frequency component is dominant within a geologic feature (Castagna and Sun, 2006). This carbonate reservoir consists of several buried channels which would certainly be interesting candidate to verify probable places of hydrocarbon accumulation. Hence, it is ideally necessary to know porosity or lithology of the channels, because channeling may enhance or decrease the porosity of the reservoir. Seismic inversion yields model of earth, represented by an acoustic or elastic impedance attribute that is sensitive to change of porosity or lithology within the layers themselves (Veeken and Da Silva, 2004). In this study, to detect the channel patterns, the FFT method selected which is sensitive to the features of the channel changes. Also acoustic impedance is integrated with the results of channel analysis to determine carbonate reservoir characteristics of them.

\section{Method and/or Theory}

In this study, the FFT method is performed using 3D-seismic volume with a time interval of $1000 \mathrm{~ms}$ in an approximate of $1050 \mathrm{~km}^{2}$. In the first step, to detect channel patterns, iso-frequency maps are directly generated with length of time window selected between 10 to $100 \mathrm{~ms}$ in frequencies of 15, 20, $25,30,35$ and $40 \mathrm{~Hz}$. These iso-frequency maps are calculated to investigate lateral variations and thickness changes in frequency contents of channel patterns. An expert interpreter could obtain some information about channel frequency contents through observed variations of the iso-frequency maps. The major problem in this study is the opposite behaviour of channels in different stratigraphic levels, when their frequency contents are compared with surrounding parts. Therefore, they are classified into two patterns based on their frequency content in comparison with their surroundings. Figure 1 shows the iso-frequency maps along two stratigraphic levels of carbonate reservoir.

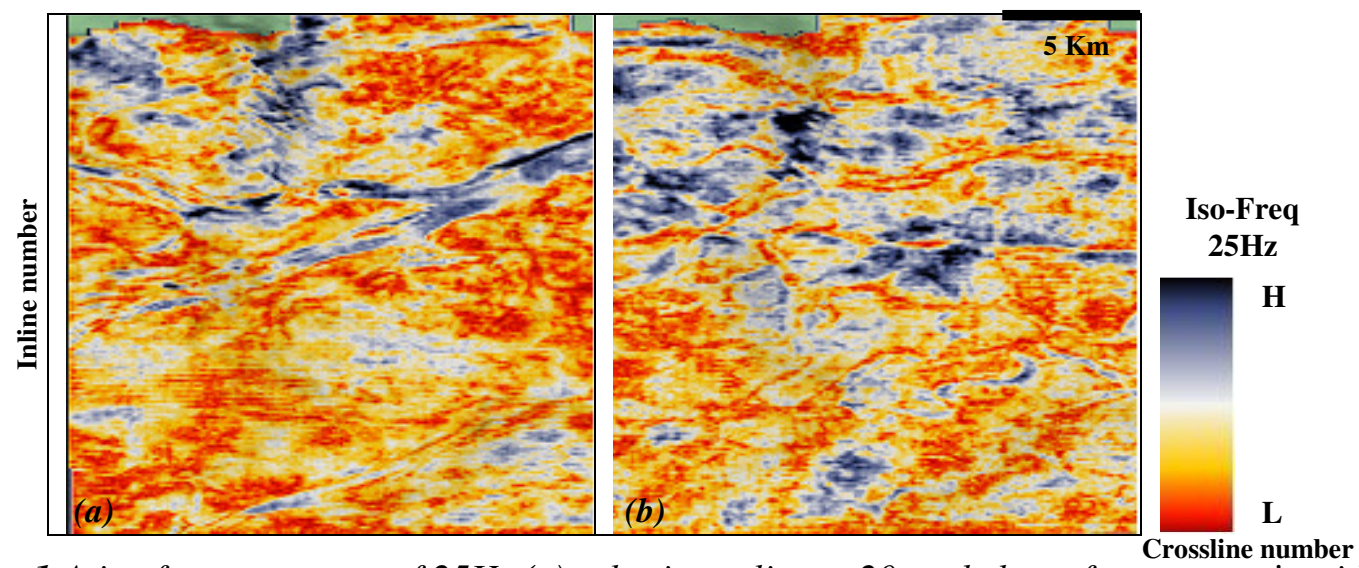

Figure 1 A iso-frequency map of $25 \mathrm{~Hz}$ (a) a horizon-slice at $20 \mathrm{~ms}$ below of top reservoir with a time window equal to $56 \mathrm{~ms}(\boldsymbol{b})$ a horizon-slice at $140 \mathrm{~ms}$ below of top reservoir and length of time window $32 \mathrm{~ms}$ (a) The frequency contents of upper channels is higher than from their surroundings, whereas (b) the frequency contents of deeper channels is lower than from their surroundings.

In the next step, to study porosity or lithology of the channels, post-stack seismic inversion is used based on model-based inversion algorithm of PSTM 3D seismic cubes, 18 wells with sonic and density logs, and interpreted seismic horizons. Quality control and pre-conditioning of the input data, 


\section{Amsterdam ' 14}

well-to-seismic tie, extraction of wavelet and low frequency model building are preformed as main steps for this study. After finalizing inversion parameters through extensive testing, derived parameters are applied for a $3 \mathrm{D}$ cube to run the inversion algorithm to obtain 3D volumes of $\mathrm{AI}$. Figure 2 exhibits acoustic impedance maps along two stratigraphic levels of carbonate reservoir.

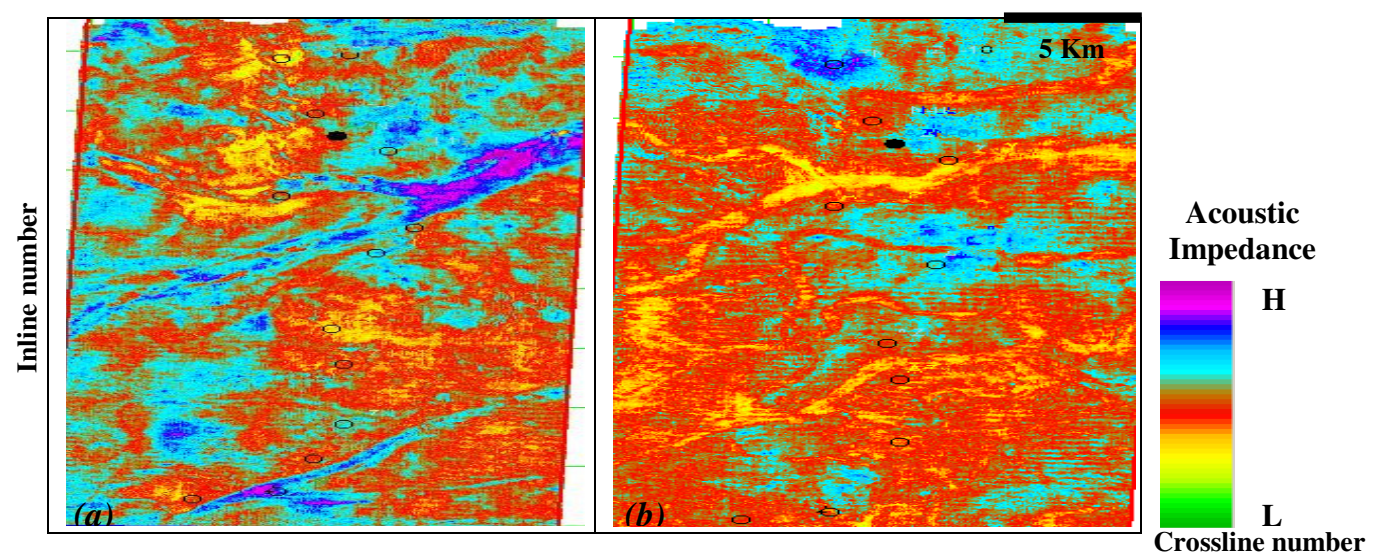

Figure 2 Acoustic impedance (a) a horizon-slice at $20 \mathrm{~ms}$ below of top reservoir, (b) a horizon-slice at $140 \mathrm{~ms}$ below of top reservoir. (a) AI in the central area of channel is higher than the eastern part and lower than the west region which means laterally varying channel properties. While, the AI of other detected channels show relatively high impedance. (b) Dipper channels have relatively low AI.

\section{Conclusions}

In this study, channels are analyzed to detect subtle changes and to determine reservoir characteristics. In application of the FFT method, the iso-frequency maps are estimated to investigate lateral variations through observed variations of the channel frequency content. The iso-frequency maps can help to clarify the channel patterns much better than the original seismic data due to low frequencies are sensitive to channel content and high frequencies are sensitive to channel boundaries. To determine reservoir characteristics of the channels, seismic inversion method is used that confirms the opposite behaviour of acoustic impedance. Deeper channels have relatively low impedance and thus sediments within them are probably more porous than surroundings. While in upper channels, the channel in central area has higher AI rather than the eastern part and lower to western region which means laterally variable channel properties. On the other hand, the AI of other detected channels suggests channel-fills are relatively tight than surroundings. As implied from low AI, the channel porosity is relatively higher than surroundings, producing relatively higher reservoir potential than surroundings. Based on the obtained results, channel patterns can be classified into two types in several stratigraphic levels of Cenomanian-Turonian carbonate reservoir based on different frequency content and acoustic impedance from their surroundings. Finally integrated analysis of iso-frequency maps with AI maps reveals porous parts of channels with more detail than single analysis of AI maps.

\section{Acknowledgments}

The authors thank NIOC Exploration Directorate for data provided in this research.

\section{References}

Castagna, J.P. and Sun, S. [2006] Comparison of spectral decomposition methods, First Break, 24, 75-79.

Hassanzadeh, A.J., Nabi-Bidhendi, M., Javaherian, A. and Pishvaiel, M.R. [2006] Integrated seismic attributes to characterize a widely distributed carbonate clastic deposit system in Khuzestan Province, SW Iran. Journal of Geophysics and Enigineering, 6, 162-171.

Veeken, P.C.H. and Da Silva, M. [2004] Seismic inversion methods and some of their constraints. First Break, 22, 47-70. 